\title{
Introducing the Media Use Behaviour Conceptual Framework
}

\author{
Douglas A. Parry ${ }^{(\bowtie)}$ (D) and Daniel B. le Roux (D) \\ Information Science, Stellenbosch University, Stellenbosch, South Africa \\ \{dougaparry, dbleroux\}@sun.ac.za
}

\begin{abstract}
In an increasingly digitally connected world researchers have sought to understand behaviour associated with digital communications media. We argue that a more consistent conceptualisation of media use behaviour and its etiological foundations is a necessary basis for research in this regard to progress. To this end, through the adoption of an affordances approach, we propose the Media Use Behaviour Conceptual Framework to describe the reciprocal relations between users (described in relation to personal characteristics and cognitive factors), the situations (consisting of social, physical, and technological dimensions) in which they use media, their media use behaviour, and the outcomes (both realised and expected) of this behaviour. This framework seeks to integrate the behaviourist and cognitivist approaches to action and, additionally, acknowledges the socially constructed and deterministic role of media in action. It is argued that such a framework will provide a useful basis upon which researchers can consider various individual differences in observed media use behaviours and associated outcomes and, importantly, understand why particular media use behaviours occur.
\end{abstract}

Keywords: Media use $\cdot$ Conceptual framework $\cdot$ Behaviour · Affordances

\section{Introduction}

Despite growing public concern and academic attention, there remains little scientific consensus on the existence, nature, and mechanisms for the effects of digital media use on psychological well-being. Mirroring the growth of social media and the widespread use of mobile computing devices, over the preceding decade, a surge of studies have sought to quantify media use and assess possible adverse associations with a variety of well-being indicators. Across this research base effects are inconsistent, generally inconclusive and, as Orben and Przybylski [22, p. 682] note, often small. While measurement artefacts present in retrospective self-report scales [6], questionable research practices [23,26], or indeed particularly nuanced relations between media use and well-being may account for disparate outcomes, the manner in which researchers conceptualise media use behaviour and its etiological foundations merits consideration as an important contributing factor to the outcomes observed.

(C) IFIP International Federation for Information Processing 2020

Published by Springer Nature Switzerland AG 2020

M. Hattingh et al. (Eds.): I3E 2020, LNCS 12067, pp. 175-186, 2020.

https://doi.org/10.1007/978-3-030-45002-1_15 
In this paper, driven by three motivations, we present a conceptual framework for media use behaviour. First, due to cycles of technological advancement the media we use have diversified rapidly and, as a result, the notion of media use has become increasingly complex. Second, extending from this complexity and the centrality of media in our lives today is the need to make sense of the agency of media use in all aspects of our lives, including, in particular, well-being. A third motivation for presenting the Media Use Behaviour Conceptual Framework acknowledges studies indicating that different types of media use are associated with a variety of effects, and, importantly, that such effects can differ across individual and situational factors [22]. A conceptual framework for media use behaviour will aid researchers in identifying specific drivers for particular media use behaviours. To follow, a description of the conceptual framework is provided, with a detailed explanation of its constituent components thereafter.

\section{A Conceptual Framework for Understanding Media Use Behaviour}

This framework, presented in Fig. 1, adopts an affordances perspective to describing interactions between individuals and the communications artefacts or media they use. Through affordances, building on an integration of behaviourist and cognitivist perspectives on behaviour, a situated conception of media use is proposed. Artefacts, consisting of both physical (hardware) and virtual (software) dimensions present various affordances which, drawing on the subjective and situational characteristics of the user, can be enacted in a variety of ways to enable particular uses and actions that, through both realised and expected outcomes, contribute to subsequent use-instances. Such a perspective enables a dualistic understanding of media use behaviour which simultaneously acknowledges the agency of technology as well as the subjective-situational dimensions of action. A medium - for instance a mobile phone - through its affordances provides a number of action possibilities for the user which, dependent on the social and physical nature of their present situation, their personal and cognitive characteristics, and previous experiences of use, can be enacted in a variety of ways. While the user has agency, this agency is constrained by the finite possibilities of action offered by the medium and the situations in which use occurs.

From a behaviourist perspective two models, both building on social learning theory, provide an initial basis for the framework. The $S O B C$ model, proposed by Davis and Luthans [4], extending earlier theories (e.g., antecedent-behaviourconsequence), postulates that aspects of an environmental situation (S) influence the internal states of an organism (O; in this case a person), which then trigger associated behavioural responses (B) and consequences (C). Building on a similar basis, emphasising the role of cognition in behaviour, the Social Cognitive Theory (SCT), [1, p. 24] proposes that behaviour occurs from the reciprocal interaction between personal (cognitive, affective, and biological), environmental, and behavioural factors. Environmental factors refer to aspects external to 


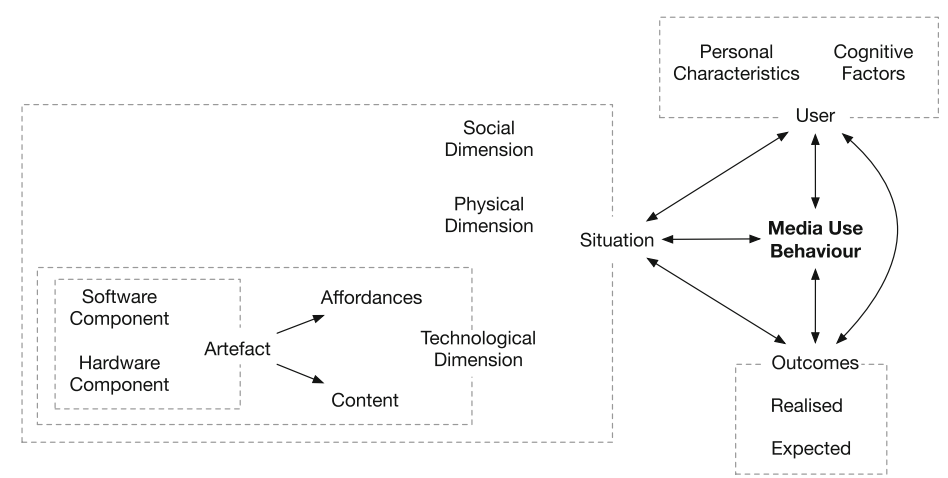

Fig. 1. Media use Behaviour Conceptual Framework

the individual which influence the ability to perform an action, behavioural factors describe the consequences resulting from an action, and personal factors relate to an individual's self-efficacy towards the behaviour. While these models recognise the mediating role of cognition, little attention is afforded to the reciprocal manner in which various executive functions and cognitive frames relate to behaviour. To provide a more holistic conception of media use behaviour, we argue that, from the perspective of situated action, affordances provide a conceptual bridge, enabling the aforementioned behaviourist models to be augmented with ideas from cognitivism (e.g., executive functions, cognitive schemas) and considerations of relevant technological factors. For each dimension of the framework, acknowledging the limited and subjective nature of the selections, we have identified appropriate conceptual theories as explanatory lenses.

\section{An Affordances Perspective}

The concept of affordances, first used by Gibson [9] to describe relations between an organism and its environment, is frequently adopted to describe the properties of an artefact - either perceived or actual - framing how it can be used. Norman [21] proposes that, through 'perceived affordances', artefacts can be designed to encourage or determine particular uses. Extending this, Gaver [7, p. 80] proposes that affordances can be both perceptible and hidden-they can be inferred - and are, as a result, 'properties of the world defined with respect to people's interaction with it'. In this way, affordances do not just apply to individual actions but, perhaps more importantly, to social interaction and the associated social conventions or cultures surrounding their enactment [8, p. 114]. While Gaver [8] avoids casting overly deterministic aspersions about the role technological affordances play in directing action, in proposing that the material (or perceptual) qualities of various communicative artefacts are, at least partly, responsible for associated actions and sociality, the foundation for the related concepts of social and communicative affordances is established. The notion of 
social affordances concerns the ways in which 'technology affords social practice' [13], while communicative affordances refers to the 'possibilities for action that emerge from [...] given technological forms' [14, p. 30]. In emphasising action possibilities this definition draws together Gibson [9]'s relational conception with the more functional conceptions of Norman and Gaver. Affordances, accordingly, enable the consideration of media use as both socially constructed and technologically deterministic [14]. Actions are enabled (and constrained) not only by the technological features and affordances of an artefact but also the subjective and situational circumstances of the user. Affordances, consequently, present a useful perspective to consider the reciprocal interactions between individuals, situations, media, media use behaviour, and behavioural outcomes.

\section{User Factors}

The framework distinguishes between two user factors. Cognitive factors describe the internal mental processes of the user, while personal characteristics refer to various traits (e.g., gender, age, personality), states (e.g., sleep, intoxication, anxiety, stress), and motivations (e.g., goals, intentions, needs). We argue that the uses of, and outcomes associated with, media are, partly, dependent on individual differences for these factors.

\subsection{Cognitive Factors}

Action, from a cognitivist perspective, occurs through internal mental processes contingent on the operation of various executive functions (e.g., working memory, inhibitory control, cognitive flexibility) [20]. Instead of the relatively simple stimulus-response relationships advanced by behaviourists, an individual is seen to play an important mediating role in these relationships. For cognitivists, an individual's internal mental processes (attention, memory, perception) are deemed to be central to behaviour. An information processing metaphor captures this notion: information is perceived, is processed by various executive functions and, depending on the stimulus and prevailing goals, behavioural responses are issued.

This information processing metaphor reflects the notion of action-oriented perception -sensory input is a function of an individual's active exploration of the world which, importantly, is framed in relation to various anticipatory schemas - clusters of related information, knowledge, or memories [20]. Stimuli are processed into schemas or cognitive frameworks of objects or events - mental frames produced through experience and knowledge. Schemas, consequently, represent recurrent patterns of embodied experience and pre-perceptual biases developed through previous interaction experiences. Acting on the basis of a schema entails the expectation of certain consequences, borne through experience. Johnson [17, p. 14] proposed the notion of embodied schemas, arguing that meaning, and the formation of meaning, stems from "patterns of embodied experience and preconceptual structures of our sensibility (i.e., our mode of perception, or orienting 
ourselves, and of interacting with other objects, events, or persons)". As we interact with the world, and the objects within it, conceptions of meaning are formed and captured in schemas. Consequently, cognition is embodied. Johnson $[17$, p. 14] further contends that, not only are schemas shaped by the artefacts we interact with but, in addition, "shared cultural modes of experience [...] help to determine the nature of our meaningful, coherent, understanding of our world". These shared cultural modes shape, and are themselves shaped by, the artifacts we use. In considering the materiality of media, the cognitive processes that direct thought and action take on a new meaning and are, themselves, dependent on various social and physical practices [17].

\subsection{Personal Characteristics}

At any point an individual's behaviour is influenced by various dispositional characteristics or traits and more momentary states. Traits, represent stable and enduring user characteristics (e.g., personality, ethnicity, cultural background, behavioural preferences). A state, in contrast, is a temporary way of being or condition (e.g., negative affect, intoxication, sleep deprivation). Importantly, while many states only manifest momentarily (i.e, in the short-term), others (e.g., mindfulness or self-control) can function as both a trait or a state and, in many cases, occur through the interaction between longer term dispositions and present situations or cognitions (e.g., self-control) [2]. Commonly referred to as individual differences, researchers frequently consider the role that various traits (physical, mental, and socially constructed) play in associations between situations, users, behaviour, and effects. Frequently analysed as moderators, such personal characteristics are seen to lead to different behavioural outcomes given the same technological affordances, depending on the individual user's assortment of traits and, in many cases, reporting of various states.

In addition to their traits and present states, users approach media use with a variety of intentions, goals, and motivations. The affordances provided by different media offer users the opportunity to gratify these needs. Uses and gratifications are, accordingly, simultaneously a function of user needs and of media affordances. The Uses and Gratifications Theory (UGT), frequently used in conjunction with the SCT, seeks to explain media use in terms of a medium's capacity to gratify a set of user needs [24]. Three assumptions are fundamental to this approach: (i) behaviour is goal-directed; (ii) users are aware of their needs; and (iii) users actively seek to gratify these needs through media use. Additionally, two distinct gratifications are advanced-process gratifications (obtained by using the media) and content gratifications (obtained from the informational content acquired through media use). Rubin [24, p. 167] considers behaviour to be largely goal-directed, arguing that "people typically choose to participate and select media [...] from an array of communication alternatives in response to their expectations and desires" which "emanate from, and are constrained by, personal traits, social context, and interaction". As noted, in addition to the needs of a user, media use behaviour is, in part, prescribed by the affordances made available by the medium. Consequently, Sundar and Limperos [29, p. 511] 
argue that, while some gratifications are driven more by user needs, others are driven more by the action possibilities of the medium.

\section{$5 \quad$ Situational Factors}

Actions do not occur in a vacuum solely extending from various schemata or motivations, nor are they mere reflexive outcomes of stimulus-response relationships with technological affordances. Rather, as in the situated action approach, along with personal characteristics and cognitions, actions are influenced by the material and social circumstances of the actor [28]. Extending Gibson [9]'s relational conception of affordances, this perspective holds that inherent qualities in the environment permit or constrain action. Importantly, while Gibson [9] advocated for a direct relationship between perception and action, this approach postulates that, rather than an unmediated process, situational affordances are mediated by individual mental models or schemata for action-thought and action are situated in physical and social contexts [11]. Cognition is situated. This conception aligns with the affordances perspective adopted. Situational affordances do not determine action but, rather, should be considered as preconditions for action, affecting the likelihood that a particular action will occur.

While, theoretically, it is straightforward to propose that action is situated, practically, researchers have struggled to settle on a widely adopted definition for what constitutes a situation. Zhang and Zhang [32, p. 1885] argue that this is due to the ontological understanding of situation as fundamentally diverse and the ethnomethodological stance that denies any a priori classifications of situational factors. Goffman [10] considers situations to be settings defined by interpersonal relationships and communication. Commenting on this Meyrowitz [19] suggests that this understanding of physical space and social situation needs to be reconsidered to account for the ability of digital media to extend communication across situations previously held to be distinct. Moreso than ever before, due to increases in the networked mobility and accessibility of mobile communications devices, social interaction occurs simultaneously through physically co-located and digitally mediated exchanges. Moreover, these developments in portability increase the number and diversity of situations in which media are used. Integrating ethnographic observations of media use in various physical locations with research considering the setting of use to be constructed by the technological artefact itself Ito and Okabe [15] proposed the concept of technosocial situations to account for the technological, social, and physical dimensions of a situation. Just as Gaver [8] avoids framing technological affordances as principle determinants of action, Ito and Okabe [15] are careful to acknowledge the continued importance of the physical and social dimensions of a situation, along with the importance of various cognitions, as enablers of action.

Situation, then, can be understood through three dimensions: the physical environment, the technological environment, and the social environment. The physical environment refers to the material properties of the setting in which media use occurs. While some media are used in relatively static locations (e.g., 
desktop computers) others are used in a large number of diverse locations (e.g., mobile phones, laptops). Different physical locations (e.g., university lectures, private bedrooms, public restaurants, open offices) present various factors which either enable or constrain particular actions. Chief among such factors are the social relationships and norms embedded within a physical location. Through various social conventions, norms, and expectations the social environment supports particular media uses, while restricting others. In specifying acceptable behaviour in a given situation, norms motivate action through promising social sanctions for counternormative conduct. Importantly, social boundaries and conventions simultaneously shape media use and are, themselves, shaped by media use [15]. Conversations on mobile phones, for instance, follow generally accepted conversational cues but are now accompanied by an expectation of continuous, uninterrupted availability. The technological dimension refers to the artefacts available in a given situation. Such artefacts determine the nature of the technological affordances available for the user to enact. Situational dimensions can, reciprocally, interact with various user, behavioural, and outcome-related factors. For instance, needs, which in turn drive various gratifications, differ from situation to situation but, as Zhang and Zhang [32] argue, situations are, themselves, influenced by various psychological needs. Similarly, an expected outcome of a particular form of media use behaviour may differ between situations.

\subsection{Artefacts}

Today, popular devices such as laptops, tablets, and smartphones provide an array of communication modes to users. In this context, two dimensions of the technological artefact merit consideration-hardware and software. Together, these dimensions determine the affordances available to a user. Hardware refers to the material, physical component of any communications artefact or medium, while software refers to the virtual component of a medium represented by the encoding of information through various programming languages and frameworks and, as perceived by the user, typically forms the graphical user interface through which interactions occur. Importantly, while differences exist, from a user's perspective, the same software can be used or accessed in the form of programs, applications, or web-services, across multiple distinct hardware forms (e.g., Facebook can be accessed on mobile phones, tablets, laptops, as a standalone mobile application, or through a web browser). The communications artefact or medium is constituted by the combination of various hardware and software characteristics. Together, this determines the nature of the affordances available to be enacted. Additionally, while media convergence increasingly implies that distinctions are difficult to make [16], the hardware and, in particular, the software components of an artefact influence the nature of content interacted with. In addition to the individual affordances of any one medium, Helles [12] argues that, owing to the increasing convergence of media, it is also necessary to account for intermediality. Referring to the interconnectedness of modern communications media, intermediality accepts that, further to the individual affordances of a particular medium, actual use frequently involves the 
combination and interrelation of multiple distinct media [16], across both hardware and software components.

Through the combination of various hardware, software, and inter-media relations, communications media present a variety of unique characteristics that, it is argued, interact with other situational dimensions, user characteristics, and outcomes, to facilitate various forms of media use behaviour. These include, but are not limited to, accessibility, flexibility, and centrality.

Extending from advances in processing power, mobility, energy storage, and information transmission, access to media, across a variety of locations and situations, is now possible. Many modern popular communications devices are designed to be portable, with batteries and wireless network connections enabling use that is untethered from physical connections to electrical power or networks. This enables the same artefact to be used across multiple distinct situations. Media are always available, always accessible, ubiquitous. Stiegler [27] proposes that the ability of users to receive and transmit information across time and space dislocates experiences from spatial and temporal contexts. Mediated interactions are reduced to a 'real-time present'. Castells [3, p. 491], accordingly, notes how media engender experiences of 'timeless time' in which both simultaneity and timelessness characterise interactions and instances of media use.

Modern communications media are highly flexible, adaptable and, given intermediality, integrated across devices, applications, and modalities. Rather than more linear modes of interaction, as in more traditional notions of mass media communication, mediated communication no longer consists of active senders and passive receivers. Rather, all users actively engage in the transmission, reception and modification of information in a manner which draws upon their existing identities, contexts and capacities. Users are free to use media in a variety of ways and for a variety of purposes, many of which were not conceived by the original producers. Considering the prominence of the World Wide Web, built on the hypertextual architecture of the Internet, as a hypermedium of communication and the manner in which modern operating systems allow the ad-hoc navigation between various programs and applications, it is argued that users are afforded a great deal of flexibility in how they can use various media. In addition to user flexibility, many media are in constant flux, with continuous release cycles prompting rapid changes in appearances and features.

Advancements in the accessibility and adaptability of media, coupled with continued developments in software capabilities and increases in the variety and richness of content made available, have enabled considerable improvements in the extent to which digital communications media can gratify a wide range of needs. Media are motivationally relevant and, consequently, central to how people work, socialise, communicate, and interact with the world around them [31]. It is argued that media are central to individuals lived-experiences to such an extent that distinctions between physical interactions and mediated interactions have become increasingly blurred. In addition to various technological affordances, the content interacted with through a medium is central to this. 
Traditionally, media use and effects have been studied in relation to the modality of communication - the medium itself - and not the content or the message, to use McLuhan [18]'s aphorism. Valkenburg et al. [30, p. 322], however, argue that, content properties cannot be ignored when seeking to understand media use behaviour and effects. Media content is typically understood to refer to the subject matter communicated by the medium (e.g., sports, news, gossip, advice, entertainment), while content properties refer to various qualities of the content (e.g., violence, aggression, outrage). Given the complexity and possible range of content-types and properties, no comprehensive theory relating media content to use behaviour, and associated effects has been produced [30]. The UGT, discussed previously, does, however, provide a useful lens to understand possible associations between media content and subsequent media use behaviour. Rubin [24, p. 167] argues that "communication behavior, including the selection and use of the [sic] media, is goal-directed, purposive, and motivated". Users actively seek out and engage with media content that gratifies their needs, desires, or goals. Traditionally, the UGT has been used to predict television viewing levels in relation to various gratifications (e.g., entertainment, information seeking) and associated content forms [25]. More recently, this approach has been used to predict specific media use behaviours in relation to categories of content accessible through various social networking services: informational, entertainment, remunerative, and relational [5]. According to the UGT, different patterns of gratifications, driven by different needs, satisfied by different media content and affordances, contribute to different behavioural outcomes. Mediated content, consequently, influences media use and, as a consequence, should not be ignored when seeking to understand various forms of media use behaviour.

\section{Behavioural Outcomes}

Media use is, typically, purposive and driven, in part, by users' needs, desires, and goals [24]. In the SCT, it is proposed that the consequences resulting from an action, in this case media use, reciprocally influence subsequent considerations of whether to perform the same action. Consequently, in the context of media use behaviour, previously realised outcomes are important drivers of subsequent media use, through expectations of similar outcomes. This draws on the UGT. Outcomes associated with interacting with media content, in the aid of gratifying associated needs, firstly, reinforce these needs and, secondly, drive users to gratify them again through similar media use behaviour. There is an expectancy that, if the same action is performed, a similar outcome will be experienced. Moreover, behavioural outcomes are key to the formation of cognitive schemas and are, as such, central to embodied cognition and the subsequent framing of action as mediated by the individual [17]. Acting on the basis of a schema entails the expectation of particular consequences, borne through experience of previous media use instances. While various effects on psycho-social well-being have been investigated [23], and are, largely inconclusive, here it is proposed, at least, media use is driven by expectations of effects associated with use. Such effects 
are, however, more closely linked with the gratification of various needs, and possible affective responses. In addition to expectations, media use behaviour, and associated outcomes, through shared patterns of enactment, shape the social, cultural, and situational norms around media use. As in the SCT, behaviour producing outcomes deemed socially normative are encouraged, while behaviour producing outcomes considered to be counternormative is discouraged.

\section{Discussion and Conclusions}

In this paper we proposed a conceptual framework for understanding media use behaviour in relation to technological artefacts, their affordances and content, a user's subjective and situational circumstances, the enactment culture emerging from repeated patterns of use, and the potential outcomes of use itself. This framework does not presume to describe all factors potentially precipitating communicative action with media. Rather, it serves to guide researchers in identifying and describing the factors needed to understand a particular instance of media use behaviour and the potential effects this behaviour may enable. In particular, the framework seeks to demonstrate the complex multi-causality that is present in the formation, enactment, and potential consequences of media use behaviour. There is, accordingly, a need to move past simple conceptions of 'screen-time' and consider media use as a complex, nuanced behaviour [22,23].

Table 1. Example application of the Media Use Behaviour Conceptual Framework.

\begin{tabular}{|c|c|c|}
\hline & Factor & Example \\
\hline \multirow[t]{4}{*}{ Artefact } & Hardware & $\begin{array}{l}\text { Which computing device is being used? (E.g., } \\
\text { smartphones, laptop computers, tablets) }\end{array}$ \\
\hline & Software & $\begin{array}{l}\text { Which platforms are being used? (E.g., WhatsApp, } \\
\text { Snapchat) }\end{array}$ \\
\hline & Affordances & $\begin{array}{l}\text { How is the platform used? (E.g., reading/sending } \\
\text { messages, updating profile/status) }\end{array}$ \\
\hline & Content & $\begin{array}{l}\text { What is the thematic nature of the messages } \\
\text { sent/received? (E.g., gossip, schoolwork) }\end{array}$ \\
\hline \multirow[t]{2}{*}{ Situation } & Physical & $\begin{array}{l}\text { Where is the user located? (E.g., home, school, public } \\
\text { space) }\end{array}$ \\
\hline & Social & Who is with the user? (E.g., friends, family, peers) \\
\hline \multirow[t]{2}{*}{ User } & Personal & $\begin{array}{l}\text { What are the personal traits/states of the media user? } \\
\text { (E.g., age, gender, personality, ethnicity, stress) }\end{array}$ \\
\hline & Cognitive & $\begin{array}{l}\text { What is the nature of the cognitive schemas held the media } \\
\text { user? (E.g., frames of reference, perceived social norms) }\end{array}$ \\
\hline \multirow[t]{2}{*}{ Outcomes } & Expected & $\begin{array}{l}\text { Which outcomes were expected before/during media use? } \\
\text { (E.g., positive feedback, mood optimisation) }\end{array}$ \\
\hline & Realised & Which outcomes actualised? (E.g., positive self-esteem) \\
\hline
\end{tabular}


As we argue with this framework, the etiological foundations of media use behaviour are, arguably, particularly complex, reciprocal, multi-causal and, specifically, highly contextual on individual and situational differences. This would suggest that, similarly, any effects of media use behaviour would share such characteristics and, as a consequence, require similarly nuanced conceptions, operationalisations, and empirical models for their assessment. It is envisaged that the framework may present as a useful tool for researchers seeking to understand why a particular form of media use behaviour occurs. This is especially the case for studies using qualitative data, where such a framework can, potentially, guide the identification and classification of various behavioural drivers. For example, when analysing an individual adolescent's participation in group-based instant messaging among a circle of friends, the framework draws the researchers' attention to the following factors depicted Table 1.

Notwithstanding the value of the framework, its theoretical basis, and the subsequent conceptual discussion presented in this paper, it is acknowledged that, as a high-level conceptual description, there exist a number of limitations to the media use behaviour conceptual framework. Specifically, as a conceptual framework, no direct empirical claims to causality are proposed, described, or assessed. Additionally, the framework is presented at an abstract level. Researchers adopting the framework to consider any specific form of media use behaviour and potentially associated effects would need to draw on other research to identify the relevant operationalisations for each of the framework's components.

\section{References}

1. Bandura, A.: Social Foundations of Thought and Action: A Social Cognitive Theory. Prentice-Hall Series in Social Learning Theory. Prentice-Hall, Upper Saddle River (1986)

2. Baumeister, R.F., Schmeichel, B., Vohs, K.: Self-regulation and the executive function: the self as controlling agent. In: Kruglanski, A., Higgins, E. (eds.) Social Psychology: Handbook of Basic Principles, Chap. 22, 2nd edn, pp. 516-539. The Guilford Press, New York City (2007)

3. Castells, M.: The Rise of the Network Society: The Information Age: Economy, Society, and Culture. Wiley, New York City (2011)

4. Davis, T.R., Luthans, F.: A social learning approach to organizational behavior. Acad. Manag. Rev. 5(2), 281-290 (1980)

5. Dolan, R., Conduit, J., Fahy, J., Goodman, S.: Social media engagement behaviour: a uses and gratifications perspective. J. Strat. Mark. 24(3-4), 261-277 (2016)

6. Ellis, D.A.: Are smartphones really that bad? Improving the psychological measurement of technology-related behaviors. Comput. Hum. Behav. 97, 60-66 (2019)

7. Gaver, W.W.: Technology affordances. In: Proceedings of the SIGCHI Conference on Human Factors in Computing Systems, pp. 79-84. ACM (1991)

8. Gaver, W.W.: Situating action II: affordances for interaction: the social is material for design. Ecol. Psychol. 8(2), 111-129 (1996)

9. Gibson, J.J.: The Ecological Approach to Visual Perception. Houghton, Mifflin and Company, Boston (1979) 
10. Goffman, E.: Behavior in Public Places; Notes on the Social Organization of Gatherings. Free Press, New York (1963)

11. Greeno, J.G.: A perspective on thinking. Am. Psychol. Assoc. 44, 134-141 (1989)

12. Helles, R.: Mobile communication and intermediality. Mob. Media Commun. 1(1), 14-19 (2013)

13. Hsieh, Y.: Online social networking skills: the social affordances approach to digital inequality. First Monday 17(4) (2012)

14. Hutchby, I.: Conversation and Technology: From the Telephone to the Internet. Wiley, Hoboken (2013)

15. Ito, M., Okabe, D.: Technosocial situations: emergent structurings of mobile Email use. Pers. Portable Pedestr. Mob. Phones Jpn. Life 20(6), 257-273 (2005)

16. Jensen, K.B.: Intermediality. In: The International Encyclopedia of Communication $(2008)$

17. Johnson, M.: The Body in the Mind: The Bodily Basis of Meaning, Imagination, and Reason. University of Chicago Press, Chicago (2013)

18. McLuhan, M.: Understanding Media: The Extensions of Man. MIT Press, Boston (1964)

19. Meyrowitz, J.: No Sense of Place: The Impact of Electronic Media on Social Behavior. Oxford University Press, Oxford (1986)

20. Neisser, U.: Cognitive Psychology. Century Psychology Series. Appleton-CenturyCrofts, Detroit (1967)

21. Norman, D.A.: The Design of Everyday Things. Basic Books, New York City (1988)

22. Orben, A., Przybylski, A.K.: Screens, teens, and psychological well-being: evidence from three time-use-diary studies. Psychol. Sci. 30(5), 682-696 (2019)

23. Orben, A., Przybylski, A.K.: The association between adolescent well-being and digital technology use. Nat. Hum. Behav. 3(2), 173-182 (2019)

24. Rubin, A.M.: Uses-and-gratifications perspective on media effects. In: Bryant, J., Oliver, M. (eds.) Media Effects: Advances in Theory and Research, pp. 165-184. Taylor \& Francis, New York (2009)

25. Rubin, A.M.: Television uses and gratifications: the interactions of viewing patterns and motivations. J. Broadcast. Electron. Media 27(1), 37-51 (1983)

26. Simmons, J.P., Nelson, L.D., Simonsohn, U.: False-positive psychology: undisclosed flexibility in data collection and analysis allows presenting anything as significant. Psychol. Sci. 22(11), 1359-1366 (2011)

27. Stiegler, B.: Technics and Time: Disorientation. Stanford University Press, San Jose (1998)

28. Suchman, L.A.: Plans and Situated Actions: The Problem of Human-Machine Communication. Learning in Doing: Social, Cognitive and Computational Perspectives. Cambridge University Press, Cambridge (1987)

29. Sundar, S., Limperos, A.: Uses and grats 2.0: new gratifications for new media. J. Broadcast. Electron. Media 57(4), 504-525 (2013)

30. Valkenburg, P.M., Peter, J., Walther, J.B.: Media effects: theory and research. Annu. Rev. Psychol. 67, 315-338 (2016)

31. Vorderer, P., Krömer, N., Schneider, F.M.: Permanently online - permanently connected: explorations into university students' use of social media and mobile smart devices. Comput. Hum. Behav. 63, 694-703 (2016)

32. Zhang, W., Zhang, L.: Explicating multitasking with computers: gratifications and situations. Comput. Hum. Behav. 28(5), 1883-1891 (2012) 